\title{
Die Bestimmungen im Paragrafen 8 des Emanzipationsedikts in Preußen bezüglich der Erlangung akademischer Lehrämter durch jüdische Wissenschaftler
}

Eine Verbesserung ihrer Stellung in allen gesellschaftlichen Lebensbereichen, so auch im öffentlichen Bildungswesen, erhofften sich viele Juden, als am 11. März 1812 das preußische Emanzipationsedikt in Kraft trat, mit dem unter anderem der Zugang von Juden zu akademischen Lehrämtern ermöglicht werden sollte. Die Hoffnungen wurden indes enttäuscht. „Man hatte den Juden alle Pforten, die in den Tempel der Wissenschaft und der geistigen Ausbildung führen, geöffnet, aber alle Pforten, die aus diesem Tempel in das Leben führen zu gedeihlicher Wirksamkeit und fröhlicher Anerkennung, verschlossen, dicht verschlossen." Mit diesen Worten bilanzierte gut dreieinhalb Jahrzehnte später der Schriftsteller und Rabbiner Ludwig Philippson im Jahr 1848, was akademisch gebildeten Juden mit dem Emanzipationsedikt in Aussicht gestellt worden war und was ihnen an tatsächlicher Integration an den Universitäten und Akademien gewährt worden war. Die Bilanz war ernüchternd.

Dabei hatten die Hoffnungen der gelehrten jüdischen Kreise weniger auf dem $\S 9$ des preußischen Emanzipationsedikts gelegen, als vielmehr auf dem $\S 8$. Der verfügte zu den Partizipationsrechten der Juden im preußischen Bildungswesen: „Sie können daher akademische Lehr- und Schul- und Gemeinde-Aemter, zu welchen sie sich geschickt gemacht haben, verwalten.“ Hier und auch „für Lehrämter" galt also das moderne, Stand und Herkunft, ignorierende Leistungsprinzip und der Leistungsnachweis, also eine Prüfung, anhand der grundsätzlich jedermann unter Beweis stellen konnte, ob und in welchem Maße er sich „geschickt gemacht“ hat. Im $\S 9$ hieß es dagegen, potentiell einschränkend, wenn auch nicht unmittelbar mit dem $\S 8$ verknüpft: „In wie fern die Juden zu andern öffentlichen Bedienungen und Staats-Aemtern zugelassen werden können, behalten wir uns vor, in der Folge der Zeit, gesetzlich zu bestimmen." ${ }^{2}$ Rechtlich bedeutete das aber angesichts der Verbindung von Lehrtätigkeiten mit dem Beamtenstatus, dass Juden der Zugang zu Staatsämtern, also zum Beispiel zu einer Beam-

1 Philippson, Ludwig: Die Theilnahme der Juden an den revolutionären Bewegungen, in: Allgemeine Zeitung des Judenthums 43 (1848), S. 617.

2 Edikt betreffend die bürgerlichen Verhältnisse der Juden im preußischen Staate vom 11. 3. 1812, Text des Edikts im Anhang dieses Bandes. 
tenlaufbahn verwehrt blieb, solange die im § 9 in Aussicht gestellte ergänzende gesetzliche Bestimmung nicht erlassen wurde. Monika Richarz stellt hierzu fest: „Das hier angekündigte Ergänzungsgesetz erschien niemals, und auch die in $\S 8$ gewährten Rechte blieben durch das Einsetzen der Reaktion fast ohne jede praktische Bedeutung. “3 Dabei hatte der $\S 8$ durchaus verheißungsvoll, ja sogar recht verbindlich geklungen.

\section{Gelehrtes jüdisches Leben im Kontext der Berliner Universitätsgründung}

Einer der Orte, auf den sich die Hoffnungen zahlreicher jüdischer Gelehrter richtete und der noch vor dem Emanzipationsedikt im Zentrum der preußischen Reformen stand, war die 1810 ins Leben gerufene Berliner Universität. ${ }^{4}$ Doch auch hier ist festzustellen, dass das gelehrte jüdische Leben Berlins in den ersten Jahrzehnten der Universität institutionell nahezu völlig ausgeklammert blieb. Das wissenschaftliche Potential, das dadurch ungenutzt blieb, war gemessen am Qualifikationsprofil, das die Zusammensetzung des ersten Lehrkörpers der jungen Universität kennzeichnete, durchaus beträchtlich. Denn die Zentren jüdischer Gelehrsamkeit in der preußischen Hauptstadt wurden zu Beginn des 19. Jahrhunderts nicht allein durch die berühmten Salons repräsentiert. Namhafte jüdische Wissenschaftler, wie Moses Mendelssohn (1729-1786), der Arzt, Philosoph und Freund Kants, Marcus Herz (1747-1803), oder der Begründer der Ichthyologie, Marcus Élesier Bloch (1723-1799), dessen bedeutende Fischsammlung 1810 in den Besitz des zur Universität gehörenden Berliner Zoologischen Museums überging, waren zwar bereits verstorben, hatten aber durch ihre über Jahrzehnte in Berlin ausgeübte Praxis des Forschens, Lehrens, Sammelns und Publizierens Grundsteine gelegt und Pionierleistungen erbracht, die die Berliner Universität in der Gestalt ihrer Gründungsidee mit ermöglicht hatten.

Im späten 18. Jahrhundert und an der Schwelle zum 19. Jahrhundert wurden in Berlin auf breiter Basis neue Organisationsformen und Vereinigungen gegründet, die eine regelrechte Bildungsoffensive innerhalb des jüdischen Lebens

3 Richarz, Monika: Der Eintritt der Juden in die Akademischen Berufe. Jüdische Studenten und Akademiker in Deutschland 1678-1848, Tübingen 1974, S. 165.

4 Vgl. im Folgenden auch meine Darstellung in: Treß, Werner: Professoren. Der Lehrkörper und seine Praxis zwischen Wissenschaft, Politik und Gesellschaft, in: Tenorth, Heinz-Elmar/ McClelland, Charles (Hrsg): Geschichte der Universität Unter den Linden, Bd. 1: Gründung und Blütezeit der Universität zu Berlin, Berlin 2012, S. 131-207, hier: S. 155-167. 
der Stadt evozierten und wissenschaftliche Betätigungsfelder nicht nur mit einschlossen, sondern ihre Akteure im forscherischen Arbeiten auch beförderten. $\mathrm{Zu}$ nennen sind hier unter anderem die seit Gründung der Freischule 1778 immer zahlreicher werdenden jüdischen Schulen ${ }^{5}$ und Vereinigungen, wie die 1783 eröffnete „Veitel-Heine-Ephraimsche Lehranstalt“6 ${ }^{\text {“6 }}$ und die 1792 gegründete „Gesellschaft der Freunde“. ${ }^{7}$ So wirkten im Kontext der Gründung der Universität in Berlin unter anderem der Mathematiker und Philosoph Lazarus Bendavid (1762-1832), der bereits als Privatdozent an der Universität Wien gelehrt hatte, bevor er 1806 als Direktor der jüdischen Freischule nach Berlin kam. Aus den Reihen der „Gesellschaft der Freunde“ zu nennen sind unter anderem die auch durch wissenschaftliche Publikationen hervorgetretenen Berliner Ärzte Ludwig Rintel (1773-1861), Abraham Bing (1769-1835) und David Oppenheimer (17531815), der unter anderem als Gutachter für Hufeland tätig war. Ebenfalls in der „Gesellschaft der Freunde“ engagiert und um 1810 in Berlin präsent waren der Vertreter der jüdischen Aufklärung und Förderer der Gebrüder Humboldt David Friedländer (1750-1834), sowie dessen Sohn Benoni Friedländer (1773-1858), der in Berlin als Privatgelehrter eine bedeutende Münz- und Autografensammlung aufgebaut hatte. Erwähnt werden muss schließlich der Philosoph und Schriftsteller Saul Ascher, der im Jahr der Berliner Universitätsgründung, nachdem er im April 1810 aufgrund seiner wiederholten Konflikte mit der preußischen Pressezensur in Berlin verhaftet worden war, im September 1810 von der Universität Halle zum Doktor der Philosophie promoviert wurde. ${ }^{8}$ Die Allgemeine LiteraturZeitung vermeldete hierzu: „Die philosophische Facultät zu Halle hat dem Hn. Saul Ascher zu Berlin wegen seiner durch verschiedene Schriften rühmlich erprobten philosophischen Kenntnisse und Talente die Doctorwürde ertheilt.“9 Es hätte nach diesem Qualifikationsnachweis also durchaus nahegelegen, die „rühmlich erprobten [...] Kenntnisse und Talente“ des „zu Berlin“ ansässigen Doktors der Philosophie, Saul Ascher, mit in das Lehrangebot der dortigen Universität einzubringen. Warum offenbar nicht einmal Ascher selbst diese Möglich-

5 Siehe u. a. Fehrs, Jörg H.: Von der Heidereutergasse zum Roseneck. Jüdische Schulen in Berlin 1712-1942, Berlin 1993.

6 Siehe Linden, Harry van der: Die Veitel Heine Ephraimsche Lehranstalt, in: Grözinger, Karl E.: Die Stiftungen der preußisch-jüdischen Hofjuweliersfamilie Ephraim und ihre Spuren in der Gegenwart, Wiesbaden 2009, S. 13-52.

7 Siehe Panwitz, Sebastian: Die Gesellschaft der Freunde 1792-1935. Berliner Juden zwischen Aufklärung und Hochfinanz, Hildesheim [u. a.] 2007.

8 Zu Ascher siehe: Best, Renate: Der Schriftsteller Saul Ascher im Spannungsfeld innerjüdischer Reformen und Frühnationalismus in Deutschland, in: Ascher, Saul: Ausgewählte Werke, Köln [u. a.] 2010, S. 7-50.

9 Zitiert nach: ebd., S. 25. 
keit in Betracht zog, lässt sich angesichts des „Empfangs“, den ihm Ordinarien wie Friedrich Karl von Savigny oder Friedrich Rühs in der Berliner Universität bereitet hätten, leicht erahnen. Die publizistischen Auseinandersetzungen, die Ascher unter anderem mit diesen beiden Judenfeinden unter den Berliner Professoren führte, erreichten spätestens 1815 mit der Veröffentlichung von Aschers Germanomanie ihren Höhepunkt. ${ }^{10}$

Anders als die bisherigen preußischen Universitäten, war die Berliner Universität im Jahr 1812 jung und verfügte noch über keine Statuten, die der vorbehaltlosen Umsetzung eines neuen Gesetzes, wie dem Emanzipationsedikt, hätten entgegenstehen können. Hinzu kam, dass Wilhelm von Humboldt als Leiter der Sektion für Kultus und Unterricht der staatbürgerlichen Gleichstellung der Juden durchaus positiv gegenüber gestanden hatte, wie man der Entstehungsgeschichte des Emanzipationsedikts entnehmen kann. Kaum beachtet worden ist nämlich bisher die zeitliche Nähe, in der Humboldt seine Denkschriften zur Gleichstellung der Juden und zur Errichtung der Berliner Universität verfasste. Vom 24. Juli 1809 datiert Humboldts berühmter Antrag auf Errichtung der Universität Berlin. Das Schriftstück, das Humboldt unmittelbar vor diesem Antrag fertiggestellt hatte, datiert vom 17. Juli 1809, also exakt eine Woche davor und es trägt den Titel Über den Entwurf zu einer neuen Konstitution für die Juden. ${ }^{11}$

Wie der Name bereits erkennen lässt, handelt es sich bei diesem Schriftstück um das Gutachten, das Humboldt in seiner Zuständigkeit als Sektionsleiter zu dem entsprechenden Gesetzesentwurf Leopold von Schroetters, des Leiters der „Section der allgemeinen Polizei“, angefertigt hatte. Gegen dessen Entwurf argumentierend, erklärte Humboldt, dass es problematisch wäre, wenn man die jüdischen Bürger von Staatsämtern ausschlösse, sie zugleich aber zu den Lehrämtern zuließe. Ein solches Vorgehen würde einerseits die Juden herabwürdigen, weil man ihnen, indem man sie von den Staatsämtern ausschlösse, weiterhin das staatliche „Mißtrauen in ihr Pflichtgefühl“ signalisiere; zugleich würde es aber auch die Lehrämter herabwürdigen, weil diese nicht als gleichrangig mit den Staatsämtern behandelt würden. Humboldt plädierte daher dafür, eine gesonderte Regelung hinsichtlich der Staats-, Lehr-, Schul- und Gemeindeämter in der neuen Konstitution für die Juden gänzlich unerwähnt zu lassen. Die Gründe entsprechen dem liberalen und antiständischen Geist der Bildungsreform, den Hum-

10 Vgl. Ascher, Saul: Germanomanie. Skizze zu einem Zeitgemälde, Berlin 1815, S. 47-54 und passim. Zum Kontext der Auseinandersetzungen siehe u. a. Lund, Hannah Lotte: Die Universität in der Stadt 1810-1840. Geselligkeit - Kultur - Politik, in: Tenorth, Heinz-Elmar/McClelland, Charles (Hrsg): Geschichte der Universität, Bd. 1, S. 325-380, hier S. 349f.

11 Humboldt, Wilhelm v.: Werke (Studienausgabe in 5 Bänden), hrsg. von Andreas Flitner und Klaus Giel, Darmstadt 2010, Bd. IV, S. 95-112. 
boldt auch sonst praktizierte und für notwendig hielt. Denn Humboldt, obwohl er die Besonderheit des Zugangs zu Staatsämtern durchaus sieht, ${ }^{12}$ wiederholt hier - einerseits und im Allgemeinen - als Begründung nur, was er auch sonst sagte: Die Berechtigung des Zugangs zu Ämtern muss für alle, die über den Nachweis der geforderten Kompetenz verfügen, eine Selbstverständlichkeit sein. Sein ablehnender Kommentar galt dann vor allem dem § 10 der Entwurfsfassung, hier sieht er den Widerspruch zu $§ 8,{ }^{13}$ und schlägt daher vor, auch diesen Paragrafen zu streichen, aber den tatsächlichen Zugang zu den Ämtern „,von der Gemeinde, den Behörden und dem Könige“ abhängig zu machen. ${ }^{14}$

Zwar konnte sich Humboldt mit seinen Empfehlungen, wie an der Fortexistenz der $\S \S 9$ und 10 der Entwurfsfassung als $\S 8$ und 9 der Endfassung des Emanzipationsediktes zu ersehen ist, nicht durchsetzen. Gleichwohl weckte der liberale Geist der von ihm auf den Weg gebrachten Universitätsgründung in Berlin berechtigte Hoffnungen. Konzentriert man also die Frage des Zugangs jüdischer Gelehrter zu akademischen Lehrämtern auf das Beispiel der Berliner Universität, so lassen sich für den Zeitraum von 1810 bis 1847, als durch das „Gesetz über die Verhältnisse der Juden“ eine neue Regelung in Kraft trat, vier exemplarische Fälle darstellen. Sie zeigen fortdauernden Ausschluss der Juden von universitären Ämtern, latenten und auch manifesten Antijudaismus bei Professoren und in der Bildungs- bzw. Staatsverwaltung sowie die Reaktionen der betroffenen jüdischen Gelehrten und Studenten.

\section{Der Fall des Mediziners Nathan Friedlaender}

Der Beginn des ersten Falls reicht noch vor das Jahr 1812 und auch vor die Gründung der Berliner Universität zurück und betrifft den Arzt der Frauen- und Kinderheilkunde Dr. Nathan Friedlaender (1778-1830). Der im schlesischen Czieschowa aufgewachsene Friedlaender hatte in Halle, Göttingen, Jena und Wien Medizin studiert und nach seiner Promotion 1805 bei Christoph Wilhelm Hufeland am Berliner Collegium medico chirurgicum die Erlaubnis erhalten „Private Vorlesungen“ zu halten. Am 8. August 1809, also kurz nachdem Humboldt seinen

12 Humboldt schreibt: „Zu Staatsämtern kann an sich nicht jeder Berechtigte gelangen, sondern es bedarf einer eignen Berufung des Staats. Hier hat also der Staat die Sache beständig in seiner Hand.“ (Humboldt, Werke, Bd. IV, S. 101f.

13 In $\S 8$ der Entwurfsfassung von Schroetter heißt es: „Alle einländischen Juden genießen gleiche bürgerliche Rechte mit den Christen, in so fern diese Ordnung keine abweichende Bestimmung enthält.“

14 Humboldt, Werke, Bd. IV, S. 107, zu §§ 9 und 10. 
Antrag auf Einrichtung der Berliner Universität an den König gerichtet hatte, bewarb sich Nathan Friedlaender auf eine Professur für Entbindungskunst. Auf sein erstes „Gesuch um Anstellung“ hin beschied ihm die Sektion, wie anderen Bewerbern auch, in ihrem Antwortschreiben, dass „die Einrichtung noch nicht nahe genug sei, um über Lehrstellen disponieren zu können. “15 Friedlaender wurde mit seiner Bewerbung also zunächst vertröstet und gebeten, sich noch zu gedulden. Das tat er auch. Ein Jahr später, am 24. August 1810, bewarb er sich erneut auf eine Professur, da die Eröffnung nunmehr ja auch kurz bevorstand. In seinem Bewerbungsschreiben bot er sogar an, in Ergänzung zu der von ihm erhofften Professur auf eigene Kosten eine zusätzliche Klinik für Geburtshilfe zu errichten. Dabei zählte Friedlaender auch ausführlich auf, auf welche Leistungen im Bereich der Armen-Geburtshilfe und Kinderheilkunde er bereits verweisen konnte:

Das Touchieren und Entbinden von Frauen in der Stadt, die meine Hilfe suchten. Es kommen in der Praxis Fälle vor, die an und für sich selten und in den öffentlichen Anstalten fast gar nicht vorkommen, als z. B. vernachlässigte Geburten. Diese in der Natur zu beobachten, und die Verfahrensart dabey kennen lernen, ist dem Geburtshülfer unentbehrlich, da diese letzte den wichtigsten Teil seiner künftigen Praxis ausmachen wird, und weder Lehrbuch noch Beschreibung ihnen hierbei Genüge leisten kann. Als nun angestellter Armen Accoucheur wird es möglich sein, diesen lehrreichen Unterricht noch weiter auszudehnen und zu vervollkommnen. ${ }^{16}$

Weder seine hohen Qualifikationen noch seine Bereitschaft zu privaten Investitionen und sein karitativer Ansatz in einem der essentiellen Bereiche der Berliner Armen-Wohlfahrtspflege konnten Nathan Friedlaender aber helfen. Nachdem seine erste Bewerbung als verfrüht zurückgewiesen worden war, beschied man ihm nunmehr, dass seine Bewerbung zu spät komme. Schleiermacher, der als Vorsitzender der Einrichtungskommission der Berliner Universität auch diesmal den Entwurf für die Absage der „Sektion für Kultus und Unterricht“ an Nathan Friedlaender verfasste, formulierte es so:

Die Section bedauere Hrn Doctor Wunsch nicht erfüllen zu können, indem eine öffentl. Lehrstelle in der medicinischen Fakultät nicht mehr vacant sei, wie ihm denn auch schon früher dazu keine Hoffnung gemacht. Sie überlasse ihm indeß ob er als Privatdocent auftreten wolle und verweise ihn für diesen Fall auf eine Bekanntmachung in der hiesigen Zeitung. “17

15 Schriftwechsel zwischen Nathan Friedlaender und der Sektion für Kultus und Unterricht, Geheimes Staatsarchiv Preußischer Kulturbesitz (GStA PK), I. HA Rep 76 Kultusministerium Va Sekt. 2, Tit. I, Nr. 2, Bd. 4 [Acta ü. d. Errichtung d. Univ. z. Berlin; Laufzeit Mai-Juli 1810], Bl. 204r-204v.

16 Ebd.

17 Ebd., Bl. 204r. 
Anders als bei anderen Bewerbern, denen die Qualifikation ausdrücklich abgesprochen wurde, überhaupt an der Universität zu lehren, wird damit Friedlaenders Kompetenz von der Sektion anerkannt und ihm die Möglichkeit eröffnet, ohne weitere Prüfungen und Leistungsnachweise, an der Universität zu lehren.

Friedlaender fügte sich dieser Absage und bot fortan an der Berliner Universität Vorlesungen als Privatdozent an. Die schon als Fortschritt, wenn auch noch nicht als Durchbruch zu wertende Bedeutung seiner Personalie besteht gleichwohl darin, dass Nathan Friedlaender zumindest als der erste jüdische Privatdozent gilt, der ab 1810 an einer preußischen Universität lehrte. Seine Lehrtätigkeit in Berlin hielt er bis 1822 aufrecht. Als er 1830 in Berlin-Schöneberg starb, war er zum Begründer einer Gelehrtenfamilie geworden. Sein 1817 geborener Sohn war der spätere Nationalökonom Carl Friedlaender, der sich 1850 an der Berliner Universität habilitieren konnte und dort dreizehn Jahre später, nämlich 1863, immerhin zum außerordentlichen Professor ernannt wurde, bevor er 1876 verstarb. Dessen Söhne wiederum waren der Vulkanologe Immanuel Friedlaender (1871-1948) sowie der Zoologe, Soziologe und Sexualforscher Benedict Friedlaender (1866-1908), der im Berlin des späten 19. Jahrhunderts als Mäzen anarchistischer und sozialistischer Zeitungen sowie der frühen Homosexuellenbewegung in Erscheinung trat, bevor er sich 1908 das Leben nahm. Ein Urenkel Nathan Friedlaenders war der Geologe Carl Gotthelf Immanuel Friedlaender (1905-1991), der bis 1969 als Professor an der Dalhousie University in Halifax/Kanada lehrte. Schon dessen Vater Immanuel hatte lange vor 1933 als institutionelle Anbindung seiner wegweisenden vulkanologischen Forschungen nicht mehr auf die Universitäten in Preußen gesetzt, sondern war in die Schweiz ausgewandert.

\section{Die Judenfeindschaft der gelehrten Welt}

Von einer intellektuellen Öffnung und Anerkennung gegenüber jüdischen Gelehrten konnte an der Berliner Universität freilich auch nach Aufnahme des Lehrbetriebes keine Rede sein. Das Beispiel der „Christlich-deutschen Tischgesellschaft“, einem elitären und nationalistischen Männerbund, der es sich zur Ehre gereichen ließ, weder Frauen noch Juden - und letztere auch dann nicht, wenn sie getauft waren - in seine Reihen aufzunehmen, belegt das nachdrücklich - denn Professoren der Universität zählten zu einer wesentlichen Mitgliedergruppe der Tischgesellschaft. ${ }^{18}$ Schaut man auf die Mitgliederlisten dieser unter

18 Zur Christlich-deutschen Tischgesellschaft siehe Nienhaus, Stefan: Geschichte der deutschen Tischgesellschaft, Tübingen 2003. 
anderem von Achim von Arnim gegründeten Vereinigung, so finden sich neben bekannten Schriftstellern, Staatsräten und Militärs auch die Namen bedeutender Ordinarien der jungen Berliner Universität, so der Philosoph und kurzzeitige Universitätsrektor Johann Gottlieb Fichte, der Theologe, Vorsitzende des Senats und spätere Rektor Friedrich Schleiermacher, der Jurist und Syndikus der Universität Karl Friedrich Eichhorn sowie der Jurist Friedrich Karl von Savigny, der wenige Wochen nachdem das Emanzipationsedikt wirksam wurde, das Amt des Rektors übernahm. Savigny, der als Begründer der historischen Rechtsschule zu den einflussreichsten preußischen Juristen zählte, verantwortete übrigens im Jahr 1816 jenes bei der Juristischen Fakultät der Berliner Universität in Auftrag gegebene Rechtsgutachten, durch das den Frankfurter Juden ihre während der napoleonischen Besatzungszeit zugesprochenen Bürgerrechte wieder aberkannt wurden.

Sein Übriges zu der mehrheitlichen antijüdischen Abwehrhaltung in der Professorenschaft tat der seit 1810 ebenfalls an der Berliner Universität lehrende Historiker Friedrich Rühs, dessen 1815 in Berlin veröffentlichte Schrift Über die Ansprüche der Juden auf das deutsche Bürgerrecht zu den radikalsten Hetzschriften gegen die Judenemanzipation zählt, die aus der Zeit vor den sogenannten Hep-Hep-Unruhen im Jahr 1819 überliefert sind. ${ }^{19}$ Rühs ging in seinen Forderungen sogar so weit, dass er für die Juden die aus dem Mittelalter bekannte Kleiderordnung wieder einführen wollte, „damit ein Deutscher, selbst sei er durch Aussehen, Verhalten und Sprache irregeführt, seinen hebräischen Feind erkenne. “20

\section{Die Gewaltübergriffe auf den jüdischen Studenten Joseph Leyser Brogi}

Wie feindselig das Klima nicht nur in der Professorenschaft, sondern auch unter den Studenten der Berliner Universität war, musste Ende 1811 und im Frühjahr 1812 der jüdische Medizinstudent Joseph Leyser Brogi erfahren. ${ }^{21}$ Der aus Posen

19 Zum historischen Kontext siehe Erb, Rainer/Bergmann, Werner: Die Nachtseite der Judenemanzipation. Der Widerstand gegen die Integration der Juden in Deutschland 1780-1860, Berlin 1989.

20 Rühs, Friedrich: Über die Ansprüche der Juden auf das deutsche Bürgerrecht, Berlin 1815, S. 32, hier zitiert nach: Bruer, Albert: Aufstieg und Untergang. Eine Geschichte der Juden in Deutschland (1750-1918), Köln [u. a.] 2006, S. 213.

21 Vgl. Henne, Thomas/Kretschmann, Carsten: Friedrich Carl von Savignys Antijudaismus und die ,Nebenpolitik‘ der Berliner Universität gegen das preußische Emanzipationsedikt von 1812. Anmerkungen zu einem berühmten Fall der Universitätsgerichtsbarkeit, in: Jahrbuch für Universitätsgeschichte, Bd. 5 (2002), S. 217-225. 
stammende Brogi wurde von seinen Kommilitonen schikaniert, weil er Jude war. Nachdem er sich mehrfach verbal gegen verschiedenste Beleidigungen zur Wehr gesetzt hatte, wurde er - so schreibt es Max Lenz in seiner Geschichte der Königlichen Friedrich-Wilhelms-Universität - ,am hellen Tage und vor aller Augen auf dem Platz vor der Universität“ von einem Studenten namens Melzer mit einer Hetzpeitsche geschlagen. ${ }^{22}$ Brogi ging daraufhin zum Rektor Johann Gottlieb Fichte, um vor der Universitätsgerichtsbarkeit zu klagen. Erstaunlicherweise fand er in dem ebenfalls für seine judenfeindliche Haltung eindeutig bekannten Fichte einen Fürsprecher. Indem Fichte den Gewaltübergriff nun vor den Senat bringen wollte, um den Täter Melzer abstrafen zu lassen, ging es ihm wahrscheinlich weniger darum, dem jüdischen Studenten Brogi zu seinem Recht zu verhelfen, als vielmehr darum, gegen die verbindungsstudentischen Umtriebe an der Universität mit ihrer Neigung zu Duellen und Ehrenhändeln, die Fichte seit seinen Erfahrungen in Jena ein Dorn im Auge waren, ein Exempel zu statuieren; gleichzeitig wollte Fichte die akademische Gerichtsbarkeit stärken und die unter anderem von Savigny favorisierte Eigengerichtsbarkeit der Studenten nicht akzeptieren. In diesem Vorhaben wurde Fichte jedoch durch den Syndikus der Universität, den Jura-Professor Friedrich von Eichhorn gebremst, der den Fall an das studentische Ehrengericht übergab. Dort wurde Melzer zu einer vergleichsweise milden Karzerhaft von vier Wochen verurteilt. Brogi indes, der Opfer des Gewaltübergriffs war, wurde ebenfalls für schuldig befunden, unter anderem weil man ihm provokantes Verhalten vorwarf, und zu 14 Tagen Karzer verurteilte.

Die Reaktion auf die Botschaft, die diese Urteile des Ehrengerichts in die Studentenschaft sandten, ließ nicht lange auf sich warten. Kurze Zeit später wurde nämlich Brogi erneut in aller Öffentlichkeit von einem weiteren Studenten namens Klaatsch mit einer Ohrfeige attackiert. Und wieder wandte sich Brogi an Fichte, der diesmal umso energischer eine Verhandlung vor dem Senat einforderte. Auch diesmal war das Urteil gegen den Gewalttäter milde. Brogi hingegen musste sich von Savigny sagen lassen, dass er „aus Rachsucht und Feigheit den Gegner denunziert habe“ und „dass er durch sein Wesen zu Händeln reize“. Syndikus Eichhorn fügte auf Drängen Schleiermachers „die Drohung hinzu, daß wenn er [also Brogi - Anm. d. Verf.] sich in Zukunft durch eigene Schuld auch nur das geringste nachsagen lasse, er die Verstoßung von der Universität zu gewärtigen habe. “23

Mit solch einem Urteil der Universitätsgerichtsbarkeit aus den Februartagen des Jahres 1812 sah sich also ein jüdischer Student der Berliner Universität

22 Lenz, Max: Geschichte der Königlichen Friedrich-Wilhelms-Universität zu Berlin, Bd. 1, Halle a. d. Saale 1910, S. 410-417, hier: S. 411.

23 Lenz, Geschichte, Bd. 1, S. 417. 
konfrontiert, wenige Tage bevor das Emanzipationsedikt wirksam werden sollte. Welche Hoffnungen auf akademisches Fortkommen sollte nun ein jüdischer Student noch haben, wenn er von seinen Mitstudenten und Professoren derartig behandelt wurde? Im Fall von Joseph Brogi lässt sich diese Frage beantworten, weil er ganz unabhängig von seinen Erlebnissen an der Berliner Universität noch ein weiteres Mal in der geschichtswissenschaftlichen Literatur auftaucht, nämlich als Mitbegründer des Berliner „Wissenschaftszirkels“ im Jahre 1816. Dieser Wissenschaftszirkel war die Vorgängerorganisation des 1819 gegründeten „Vereins für Cultur und Wissenschaft der Juden“, in deren Tradition wiederum die 1872 in Berlin gegründete „Hochschule für die Wissenschaft des Judentums“ zu sehen ist.

Neben Joseph Brogi bestand 1816 der Großteil der Gründer des Berliner „Wissenschaftszirkels“ aus jüdischen Studenten der Berliner Universität. Dort eingeschrieben waren nämlich auch Leopold Zunz, Immanuel Wohlwill, Isaak Markus Jost, Julius Robo, Daniel Lessmann und Eduard Gans. Sie alle teilten die Erfahrung der strukturellen und persönlichen Diskriminierung innerhalb der Universität und gründeten daher eine private, jüdische, wissenschaftliche Einrichtung außerhalb der Universität. Trotz aller wissenschaftlichen Verdienste, die der „Wissenschaftszirkel“, der „Verein für Cultur und Wissenschaft der Juden“ und die spätere „Hochschule für die Wissenschaft des Judentums“ für sich verbuchen konnten, blieb doch der private Charakter dieser Einrichtungen immer ein Beleg für die Ausgrenzung sowohl der jüdischen Wissenschaftler als auch der Wissenschaft des Judentums durch die deutschen Universitäten und namentlich auch durch die Berliner Universität.

\section{Eduard Gans und sein Kampf um ein akademisches Lehramt}

Eine bezeichnende Ausnahme aus dem Kreis der Gründer des „Wissenschaftszirkels“ und des „Vereins für Cultur und Wissenschaft der Juden“ bildet die Geschichte der akademischen Biografie des Juristen und Hegel-Schülers Eduard Gans (1798-1839). ${ }^{24} 1816$ hatte Gans in Berlin begonnen, Jura zu studieren. Nach einem weiteren Studienaufenthalt in Göttingen promovierte er im März 1819 in Heidelberg mit einer Arbeit über „Römisches Obligationsrecht“ mit der Note „summis honoribus“. Im selben Jahr kehrte Gans nach Berlin zurück, wo er zu

$24 \mathrm{Zu}$ Gans siehe u. a. Reissner, Hans Günther: Eduard Gans. Ein Leben im Vormärz, Tübingen 1965. 
einem eifrigen Hörer der Vorlesungen Hegels wurde, dessen Werke er später mit edierte. Am 9. Dezember 1819 sandte Gans seine Bewerbung um ein akademisches Lehramt an das preußische Kultusministerium. Sein nun einsetzender Kampf um eine Professur füllt heute im „Geheimes Staatsarchiv Preußischer Kulturbesitz“ zwei Akten mit insgesamt 299 Blättern. ${ }^{25}$ Danach forderte Kultusminister Karl vom Stein zum Altenstein zunächst die juristische Fakultät der Berliner Universität auf, sich zur Bewerbung von Gans zu äußern. Erst nach mehreren Monaten sandte die Fakultät daraufhin am 4. April 1820 ein von Schmalz, Savigny, Sprickmann und Biener gezeichnetes Gutachten, dessen insgesamt negativer Tenor mit den eindeutig antijüdischen Worten endete: „Schließlich bemerken wir, daß wir nicht wissen, ob der Dr. Gans, der zu einer bekannten jüdischen Familie gehört, persönlich zur christlichen Kirche übergetreten ist, und ob also von dieser Seite kein Hinderniß mehr für seine öffentliche Anstellung vorhanden seyn mag. “26 Die Juristische Fakultät definierte hier also selbst, was in den Paragrafen 8 und 9 des Emanzipationsedikts als geltendes Recht verfügt, aber nicht präzise ausgelegt worden war.

Am 5. Juli 1820 wandte sich daraufhin der Staatskanzler Hardenberg - ein mächtiger Verbündeter für Gans - schriftlich an seinen Kabinettskollegen, den Kultusminister Altenstein, und schlug ihm vor, Eduard Gans als Privatdozent an die Universität Breslau zu schicken, ihn dort habilitieren zu lassen und aufgrund seiner gefragten Vorlesungen und breiten Kenntnisse baldmöglichst zum „Professor extraordinarius“ zu befördern. Altenstein widersetzte sich dem Ansinnen Hardenbergs und antwortete ihm mit Verweis auf das Gutachten der Juristischen Fakultät:

\begin{abstract}
Auch scheint mir der Umstand nicht unwichtig zu seyn, daß Gans, aus einer jüdischen Familie stammend, so viel wie bekannt, zum Christenthum nicht übergetreten ist. Wenn dieser Umstand auch kein rechtliches Hinderniß zu seiner Beförderung zu einem akademischen Lehramte seÿn sollte, so darf ich doch gehorsamt bemerken, daß bis dahin kein Exempel der Berufung eines Juden zum Professor der Rechte in unserm Staate statt gefunden hat, und daß die Aufnahme eine Juden in die Fakultät der Rechte, die in Beziehung auf die religiösen und politischen Verhältnisse von so großer Bedeutung ist, doch wohl einem Bedenken unterworfen seÿn dürfte. ${ }^{27}$
\end{abstract}

Das unkritische Selbstverständnis, mit dem Altenstein hier versuchte, den Geltungsanspruch der seit 1812 bestehenden Rechtslage durch Formulierungen nach der Art „so darf ich doch gehorsamst bemerken“ wegzuargumentieren, dürfte

25 GStA PK, I. HA Rep 76 Kultusministerium Vf Lit. G Nr. 2, Acta betreffend den Doctor Eduard Gans, gegenwärtig Professor bei der Universitaet zu Berlin, Bd. 1: Februar 1819-Dezember 1831. 26 Ebd., Bl. 5.

27 Ebd., Bl. 10. 
zugleich ein mustergültiges Beispiel für die Mentalität und Praxis der strukturkonservativen Beharrungskräfte in preußischen Institutionen gewesen sein. Dabei waren es gerade die Ausprägungen derart klandestiner Praktiken in den Institutionen, mit denen auch in den folgenden Jahrzehnten bis ins 20. Jahrhundert hinein die Diskriminierung jüdischer Gelehrter beim Zugang zu universitären Lehrämtern, speziell zu ordentlichen Professuren, nachhaltig behindert oder zumindest verzögert wurde.

Immerhin erkannte Altenstein in seinem Schreiben an Hardenberg an, dass der Umstand, dass Gans ein Jude sei, grundsätzlich kein rechtliches Hindernis zu seiner Berufung darstelle. Dieses Eingeständnis relativierte Altenstein jedoch in seinem Schreiben vom 16. August 1820 an Hardenberg, indem er eine Verschränkung des Charakters von staatlichen Ämtern und akademischen Lehrämtern konstruierte. Er strapazierte damit ein Argument, das seit der Demagogenverfolgung in Preußen mit Blick auf die Kontrolle der Hochschullehrer üblich geworden war, wonach nämlich den Hochschullehrern als Beamten gegen alle Zugeständnisse der Freiheit der Wissenschaften und der Universitäten besondere Pflichten und Treue gegenüber dem Staat auferlegt seien:

So scheinen ihnen auch, da Juden öffentliche Ämter nicht bekleiden können, keine Stellen übertragen werden zu dürfen, mit denen die Ausübung einer öffentlichen amtlichen Autorität verbunden ist. Dazu gehören nun nicht nur die Würden eines Rectors und Decans auf Universitäten und des Rectors an anderen gelehrten Schulen, sondern m. E. überhaupt die Ordinariate aller Facultäten, [.....$^{28}$

Diese Auslegung widersprach nun vollkommen dem Geist des Emanzipationsedikts von 1812, wonach in den $\S \S 8$ und 9 zwischen akademischen Lehrämtern und staatlichen Ämtern unterschieden wurde, obgleich Wilhelm von Humboldt wie erwähnt in seinem Gutachten von 1809 vor den Schwierigkeiten einer solchen Unterscheidung gewarnt hatte.

Nach weiteren Verzögerungen, die das Kultusministerium $\mathrm{zu}$ verantworten hatte, wandte sich Gans am 3. Mai 1821 mit resignierten Worten erneut an Hardenberg:

Ich gehöre zu der unglücklichen Menschenclasse, die man hasst, weil sie ungebildet ist, und die man verfolgt, weil sie sich bildet. So muß auch ich schon zwei Jahre ein Opfer dieses Zirkelschlusses seyn, und, wenn ich es durchaus bedauern soll, daß mein verstorbener Vater mir eine sorgfältige Erziehung reichen ließ, und daß ich mich, gegen den Wunsch meiner Familie [...] den Wissenschaften, zu denen ich meinen Beruf fühlte, ergab, so ist die beste Einleitung dazu, durch zweijährige Verfolgung, Kränkung und Zurücksetzung geschehen. Eure Excellenz haben mich mündlich höchstdero Gnade und Gewogenheit zu versichern

28 Ebd., Bl. 19. 
geruht. Ich nehme dieselbe jetzt in Anspruch nicht, um eine unverdiente Gunst zu erflehen [...], sondern um zu bitten, daß diejenige strenge Gerechtigkeit, die Ew. Excellenz keinem versage, auch mir in so kurzer Frist als möglich gewährt werde. ${ }^{29}$

Dieses Schreiben nahm Hardenberg zum Anlass, sich nochmals und diesmal mit mehr Nachdruck an Altenstein zu wenden, indem er ihn ausdrücklich auf das Edikt vom 12. März 1812 hinwies und nur mehr die Befolgung von Gesetzen durch eine Behörde einforderte:

[...] es kommt jetzt vielmehr lediglich darauf an, ein von Seiner Majestät dem Könige gegebenes Gesetz zu befolgen. Die jüdischen Unterthanen Seiner Majestät des Königs haben das gegründete Recht, solange die Verordnung von Allerhöchstdemselben nicht aufgehoben ist, deren traute Erfüllung von den Königlichen Behörden, die dazu am Schluße des Gesetzes noch ausdrücklich verpflichtet werden, zu fordern und es dürfte auf die Erhaltung der Ordnung im Allgemeinen nachteilig wirken, wenn irgend jemand, namentlich aber die höchsten Staatsbeamten von jener Erfüllung sich dispensieren. ${ }^{30}$

In diesem sich über drei Jahre hinziehenden Streit zwischen den Beteiligten, vor allem zwischen Hardenberg und Altenstein, wurde schließlich der König selbst durch Hardenberg mit dem Vorgang konfrontiert. Am 18. August 1822 erließ Friedrich Wilhelm III. daraufhin eine Kabinettsordre an den Staatskanzler Hardenberg, die als „Lex Gans“ in die Universitätsgeschichte einging. In dieser Kabinettsordre - und das entsprach gar nicht Gans' Erwartungen und dem Vorgehen und den Argumenten von Hardenberg - wurden die Zugeständnisse aus dem $\S 8$ des Emanzipationsediktes nämlich nicht bekräftigt, sondern vollständig zurückgenommen. Der Wortlaut der Kabinettsordre lässt darauf schließen, dass der König sich den Aktenlauf zu Gans genauer angeschaut hat, denn dort steht:

\footnotetext{
Aus den zurückgehenden Anlagen ihrer Anzeige vom 4. d. M. habe ich ersehen, dass die Bestimmung der $\S 7$ und 8 des Edikts vom 11. März 1812, nach welcher die für Inländer zu achtenden Juden zu akademischen Lehr- und Schulämtern, zu welchen sie sich geschickt gemacht haben, zugelassen werden sollten, nicht, ohne große Mißverhältnisse zu veranlassen, durchzuführen ist. Ich will daher diese Bestimmung hierdurch aufheben und Ihnen die weitere Verfügung wegen der Bekanntmachung dieser Abänderung des gedachten Gesetzes anheim geben. ${ }^{31}$
}

29 Ebd., Bl. 30

30 Ebd., Bl. 50

31 Zitiert nach: Kalisch, Moritz: Die Judenfrage in ihrer wahren Bedeutung für Preußen, Berlin 1860, S. 32. 
$\mathrm{Zu}$ Recht beklagte sich Gans nun darüber, dass er über Jahre „durch illusorische Gesetze“ - wie er es nannte - getäuscht und so zum „unglücklichsten Menschen“ gemacht worden sei. Noch bevor Hardenberg im Herbst 1822 zum Kongress der europäischen Staatsmänner nach Verona aufbrach, von dem er nicht mehr lebend zurückkehrte, verfügte er, dass Gans als Entschädigung ein zweijähriges Reisestipendium erhalten solle. Dieses wurde ihm 1823 durch den Kultusminister Altenstein auch tatsächlich gewährt. In der Folgezeit schwankte Gans zwischen Auswanderung und Taufe. 1825 konvertierte er schließlich zum christlichen Glauben und wurde Mitglied der Evangelischen Kirche. Nach seiner Taufe und erneuten Bewerbung unterstützte nunmehr auch Altenstein die Ernennung von Gans zum außerordentlichen Professor. Am 1. März 1826 schrieb Altenstein die entsprechende Vorlage an den König:

Nachdem Dr. Gans nach einem jahrelangen Kampfe mit sich selbst und seinen Verhältnissen endlich aus innerer Überzeugung aus freiem Antriebe das Judenthum verlassen und die göttlichen Lehren des Christenthums bekennt und die heilige Taufe empfangen hat, müssen nun durch seine Kenntnisse und ausgezeichneten Fähigkeiten im Dienste Ew. Königlichen Majestät als akademischer Universitätslehrer möglich werden zu können \& er hat in pflichtschuldiger Dankbarkeit um die Wohlthaten, welche Ew. Königliche Majestät ihm bisher huldreichst haben angedeihen lassen, vorteilhafte Anträge abgelehnt, die ihm vom Ausland her bisher [gemacht] worden sind. ${ }^{32}$

Zwölf Tage später, am 13. März 1826, erhielt Gans endlich seine Bestallungsurkunde als außerordentlicher Professor, 1828 avancierte er zum ordentlichen Professor und wurde 1832 sogar Dekan der juristischen Fakultät. Eduard Gans starb 1839.

Das außergewöhnliche an diesem Kampf, den Eduard Gans um die Erringung seiner Professur führte, war, dass er solange am jüdischen Glauben festhielt. Dass er schließlich nach seiner Taufe relativ unkompliziert zum Ordinarius avancierte, war dahingegen nichts Ungewöhnliches mehr. Unter den Ordinarien an der Berliner Universität vor 1847 war es zum Beispiel der Theologe August Neander, der 1789 mit dem Namen David Mendel geboren wurde, sich 1806 taufen ließ und 1813 als ordentlicher Professor von Heidelberg nach Berlin berufen wurde. Gleiches gilt für den Physiker Gustav Magnus, der ebenfalls bereits getauft war, bevor er 1834 seine Berliner Professur erhielt, ebenso Friedrich Julius Stahl, der, 1802 mit dem Namen Julius Jolson geboren, sich 1819 taufen ließ, bevor er 1832 zunächst in Erlangen Professor wurde und dann 1840 auf persönlichen Wunsch Friedrich Wilhelms IV. nach Berlin berufen wurde.

32 GStA PK, I. HA Rep 76 Kultusministerium Vf Lit. G Nr. 2, Bl. 131. 


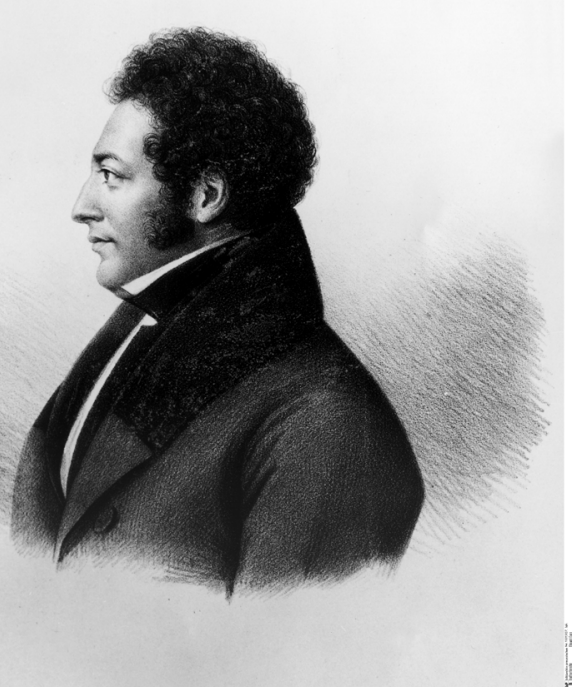

Abb. 6: Eduard Gans, Lithografie, um 1830.

\section{Die Habilitation von Robert Remak und der legislative Wandel der Emanzipationsgesetze von 1847 und 1869}

Von einem fast noch entschlosseneren Festhalten an seiner jüdischen Identität geprägt war hingegen das Ringen des jüdischen Arztes Robert Remak (1815-1865) um seine Habilitation. ${ }^{33}$ Seit seiner Promotion 1838 bemühte er sich vergebens um eine akademische Laufbahn. Am 24. Februar 1847 schrieb Remak schließlich auf Vermittlung Alexander von Humboldts an Friedrich Wilhelm IV.: „Der Uebertritt zum Christenthum wurde mir durch die Orthodoxie meiner Eltern, denen ich Pietät und Liebe schulde, unmöglich gemacht. Auch habe ich den größten Widerwillen gegen den Gedanken, durch Religionswechsel aeussere Vorteile zu erlangen.“34 Durch eine „besondere Dispensation“, wie es hieß, wurde daraufhin

33 Zum Fall von Robert Remak siehe Schmiedebach, Heinz-Peter: Jüdisches Bekenntnis und emanzipierte Wissenschaft im Vormärz. Die Habilitationsbemühungen des Arztes Robert Remak (1815-1865) an der Berliner Universität, in: Menora. Jahrbuch für deutsch-jüdische Geschichte, Bd. 4 (1993), S. 176-200.

34 Zitiert nach: ebd., S. 191. 
im März 1847 die Habilitation von Robert Remak genehmigt, ohne dass er zuvor zum Christentum konvertieren musste. Es dauerte allerdings nochmals über zehn Jahre, bis er 1859 an der Berliner Charité wenigstens zum Extraordinarius ernannt wurde.

Im selben Jahr, in dem Remak seine Zulassung zur Habilitation erhielt, wurde vom preußischen Landtag ein neues „Gesetz über die Verhältnisse der Juden“ verabschiedet. Dieses Gesetz vom 23. Juli 1847 hob die Beschränkungen der Kabinettsordre von 1822 wieder auf und konkretisierte das, was im $\S 8$ des Emanzipationsediktes von 1812 eigentlich schon beschlossen worden war. Jedoch gab es auch hier immer noch Einschränkungen:

Bei den Universitäten können Juden, soweit die Statuten nicht entgegen stehen, als Privatdozenten, außerordentliche und ordentliche Professoren der medizinischen, mathematischen, naturwissenschaftlichen, geographischen und sprachwissenschaftlichen Lehrfächer zugelassen werden. Von allen übrigen Lehrfächern an Universitäten, sowie von dem akademischen Senate und von den Ämtern eines Dekans, Pro-Dekans und Rektors bleiben sie ausgeschlossen. ${ }^{35}$

Die Ausnahmen, die dieses Gesetz nach Fakultäten und für akademische Ämter festlegte, sorgten weiterhin für eine erhebliche Rechtsunsicherheit für jüdische Gelehrte, die eine akademische Laufbahn anstrebten. Frappierend war vor allem, dass an den juristischen Fakultäten der preußischen Universitäten und damit auch in Berlin weiterhin die Berufung jüdischer Rechtswissenschaftler zum Ordinarius ausgeschlossen blieb. Die vollständige rechtliche Gleichstellung der in Preußen lebenden Juden und damit auch ihr Anrecht auf den Zugang zu ordentlichen Professuren wurde dann im sogenannten Emanzipationsgesetz des Norddeutschen Bundes vom 3. Juli 1869 geregelt. Darin hieß es in einem einzigen bündigen Artikel:

Alle noch bestehenden, aus der Verschiedenheit des religiösen Bekenntnisses hergeleiteten Beschränkungen der bürgerlichen und staatsbürgerlichen Rechte werden hierdurch aufgehoben. Insbesondere soll die Befähigung zur Theilnahme an der Gemeinde- und Landesvertretung und zur Bekleidung öffentlicher Ämter vom religiösen Bekenntniß unabhängig sein. $^{36}$

35 Gesetz über die Verhältnisse der Juden vom 23. Juli 1847, § 2, in: Gesetz-Sammlung für die Königlich Preußischen Staaten 1847, Nr. 2871, S. 263-278, hier S. 263. Siehe auch Kalisch, Judenfrage, S. 83.

36 Gesetz betreffend die Gleichberechtigung der Konfessionen in bürgerlicher und staatsbürgerlicher Beziehung vom 3. Juli 1869, in: Bundes-Gesetzblatt des Norddeutschen Bundes 1869, S. 292. 
Damit war der Prozess der staatsbürgerlichen Gleichstellung der Juden in Preußen und ab 1871 auch im Deutschen Reich auf der legislativen Ebene abgeschlossen. Dafür, dass sich gegen diese legislativen Vorgaben dann auf der institutionellstrukturellen Ebene Widerstand regte und sich eine gleichsam klandestine Praxis ihrer Nichtanwendung herausbildete, zeugte nicht nur nach dem Emanzipationsgesetz von 1812, sondern auch nach den Emanzipationsgesetzen von 1847 und 1869 eine Berufungspolitik an den preußischen bzw. deutschen Universitäten, bei der jüdische Gelehrte weiterhin ausgegrenzt blieben und nur in Ausnahmefällen auf ordentliche Professuren berufen wurden. Im Falle der Emanzipationsgesetze von 1812 und 1869 lässt sich sogar die These vertreten, dass in den jeweiligen Dekaden nach Erlass dieser Gesetze die Judenfeindschaft in der Gesellschaft sogar noch an Schärfe zunahm. Ausgangspunkte für das Auftreten eines organisierten Antisemitismus waren dabei immer auch die Universitäten, denkt man etwa an die erhebliche Deutungs- und Wirkmacht, die die Schriften der Berliner Ordinarien Friedrich Rühs Über die Ansprüche der Juden auf das deutsche Bürgerrecht (1815/1816) und Heinrich von Treitschke Unsere Ansichten (1879) auf die öffentlichen Debatten und das gesellschaftliche Klima ihrer Zeit ausübten.

Der Kampf jüdischer Wissenschaftler um den Zugang zu akademischen Lehrämtern gestaltete sich dabei oft als ein jahrzehntelanger Prozess zähen Ringens, der sich bei vielen jüdischen Gelehrtenfamilien - wie am Beispiel der Familie Friedlaender gezeigt wurde - über mehrere Generationen erstreckte. Die ganze Tragik dieses generationenübergreifenden Ringens wird auch am Beispiel der Familie Remak sichtbar. Der Arzt und Physiologe Robert Remak, dessen Bemühen um Zulassung zur Habilitation und Ernennung zum außerplanmäßigen Professor skizziert wurde, verstarb 1865 im Alter von 50 Jahren erschöpft, krank und verbittert bei einem Kuraufenthalt in Bad Kissingen. ${ }^{37}$ Sein Sohn war der bedeutende Neurologe Ernst Julius Remak, geboren 1849, der sich 1877 in Berlin habilitierte und nach 25 Jahren wissenschaftlicher und medizinischer Arbeit als Privatdozent an der Charité und an seiner privaten Poliklinik schließlich 1902 zum außerordentlichen Professor ernannt wurde. ${ }^{38}$ Er verstarb 1911 in Berlin kurz vor Vollendung seines 62. Lebensjahrs und wurde neben seinem Vater auf dem Jüdischen Friedhof in der Berliner Schönhauser Allee beerdigt. Der Sohn von Ernst Julius Remak war der nach seinem Großvater benannte Mathematiker und Nationalökonom Robert Erich Remak, geboren 1888, der nach mehreren vergeblichen Ver-

37 Vgl. Schmiedebach, Jüdisches Bekenntnis und emanzipierte Wissenschaft im Vormärz, S. 181.

38 Vgl. Holdorf, Bernd: Die nervenärztlichen Polikliniken in Berlin vor und nach 1900, in: ders./Winau, Rolf (Hrsg.): Geschichte der Neurologie in Berlin, Berlin 2000, S. 128. Den Professorentitel durfte Ernst Julius Remak seit 1893 führen. 
suchen 1929 in Berlin habilitieren konnte und als Privatdozent an der Berliner Universität lehrte und forschte, bis ihm 1933, nach dem Machtantritt der Nationalsozialisten, auf Grundlage des „Gesetzes zur Wiederherstellung des Berufsbeamtentums“ vom 7. April 1933 die Lehrbefugnis entzogen wurde. Die richtungweisende Bedeutung seiner zwischen 1929 und 1933 veröffentlichten Arbeiten für die „moderne Aktivitätsanalyse“ auf dem Gebiet der mathematischen Ökonomie wurde erst in den 1960er-Jahren von der internationalen Forschung wiederentdeckt und seither rezipiert. ${ }^{39}$ Das Leben von Robert Erich Remak endete 1942 im Vernichtungslager Auschwitz. ${ }^{40}$

39 Vgl. Hagemann, Harald: Remak, Robert, in: Neue Deutsche Biographie, Bd. 21 (2003), S. 411-412.

40 Auf dem „Stolperstein“ vor der Manteuffelstraße 22a in Berlin-Lichterfelde steht: „Hier wohnte Dr. Robert Remak, Jg. 1888, verhaftet 1938 Sachsenhausen, Flucht 1939 Holland, interniert Westerborg, deportiert 1942, ermordet in Auschwitz“. Siehe auch Bundesarchiv, Gedenkbuch. Opfer der Verfolgung der Juden unter der nationalsozialistischen Gewaltherrschaft in Deutschland 1933-1945, online unter: http://www.bundesarchiv.de/gedenkbuch, im Namenverzeichnis unter: Remak, Robert Erich. 